\title{
Cleaning the Flue Gases of Thermal Coal Power Plants from Sulfur and Nitrogen Oxides
}

\author{
Shilyaev Mihail I. \\ Tomsk State University of \\ Architecture and Building \\ Tomsk, Russian Federation \\ shmi@mail.tomsknet.ru
}

\author{
Bogomolov Alexandr R. \\ Kutateladeze Institute of \\ Thermophysics SB RAS \\ Novosibirsk, Russian Federation \\ barom@kuzstu.ru
}

\author{
Dvorovenko Igor V., Sysolyatin \\ Andrey S., Kryukov Sergey V., \\ Chemakin Maksim A. \\ T. F. Gorbachev Kuzbass State \\ Technical University \\ Kemerovo, Russian Federation \\ adiv.pmh@kuzstu.ru
}

\begin{abstract}
An experimental setup for adapting the absorption mathematical model (M.I. Shilyaev) in the Venturi scrubber for finding the phase equilibrium coefficients by debugging the algorithm in absorption processes of $\mathrm{SO} 2$ extraction by water was developed. The elements of basic equipment were calculated: Venturi scrubber (tube) and cyclone. According to the developed technological scheme of the experimental setup, the operation principle of the applied research methodology was worked out by testing $\mathrm{CO} 2$ capture by water. These tests showed an adequate operation of the Venturi scrubber in the cascade of droplet separator - cyclone. An approach to adaptation of the absorption mathematical model (AMM) for the chemisorption processes is presented. The proposed algorithm for solving the inverse problem by AMM at the example of $\mathrm{SO2}$ absorption by water in the model Venturi tube is numerically justified and can be used for experimental determination of the unknown phase equilibrium coefficients of the absorption process for all absorbent-absorbate pairs in the laboratory. Experiments on carbon dioxide capture showed the possibility of using the setup for adaptation of chemisorption processes.
\end{abstract}

Keywords: absorption, Venturi scrubber, sulfur and nitrogen oxides, absorbent, absorbate, absorption mathematical model, chemisorption processes

\section{INTRODUCTION}

At the present stage of human development, more than 850 mln tons of nitrogen oxides, $150 \mathrm{mln}$ tons of sulfur dioxide, $310 \mathrm{mln}$ tons of carbon oxide, $250 \mathrm{mln}$ tons of carbon dioxide, and $3 \mathrm{mln}$ tons of hydrocarbons are emitted annually in the atmosphere worldwide [1].

Energy companies supply to the atmosphere more than $23 \%$ of emissions from all stationary sources in the Russian Federation. Among these emissions, the share of nitrogen oxides accounts for about $97-98 \%$ (vol.), and the remaining fractions are sulfur oxides and other substances. Among sulfur oxides, the share of $\mathrm{SO}_{2}$ accounts for $97-98 \%$, another part is $\mathrm{SO}_{3}[2]$.

Since there is sulfur in the fuel, the flue gases always include sulfur dioxide. Direct interconnection between the presence of sulfur dioxide in the atmosphere and the level of human morbidity is proved.

Nitrogen and sulfur oxides contained in the flue gases are the toxic substances, and in the case of their mixing with air moisture they form acids which falling to earth in the form of the so-called acid rains damage farmland, destroy engineering structures, harm human health, and all living things on the Earth. Therefore, the development of technological processes for cleaning gas emission of TPP from harmful gas components and fine solid suspensions is a very important problem.

\section{MATERIAL AND METHODS}

The main methods used for gas emission cleaning are absorption, adsorption and catalytic method, which have their own advantages and disadvantages.

Industrial gases are emitted into the atmosphere in large quantities, sometimes exceeding a million cubic meters per hour. The current cleaning devices cannot be used because of the low capacity by gas. The only type of devices ensuring the defined cleaning requirements are the hollow vortex devices with high capacity, small size and low flow resistance, such as the Venturi scrubbers (tubes) and hollow nozzle absorbers [3] [5].

The turbulent Venturi scrubbers are one of the most effective devices for wet-type gas cleaning. The main advantages of Venturi scrubbers are easy manufacturing, installation and maintenance, small sizes and high efficiency of fine particles catching [6]. In devices of this type, cooling, humidification and absorption of gases can be carried out simultaneously with purification.

For quite a long time, the following absorbents were used in practice for sulfur oxide removal: water, water solutions of $\mathrm{Na}_{2} \mathrm{SO}_{3}(18-25 \%), \mathrm{NH}_{4} \mathrm{OH}(5-15 \%), \mathrm{Ca}(\mathrm{OH})_{2}, \mathrm{Na}_{2} \mathrm{CO}_{3}, \mathrm{NaOH}$ (15-20\%), KOH, $\left(\mathrm{NH}_{4}\right)_{2} \mathrm{SO}_{3}$ (15-20\%), $\left(\mathrm{NH}_{2}\right)_{2} \mathrm{CO}, \mathrm{ZnSO}_{4}$, $\mathrm{K}_{2} \mathrm{CO}_{3}$, etc. [4] [7].

Recently, to improve the efficiency of flue gas cleaning, the method of selective non-catalytic reduction (SNCR) [8] and absorption of $\mathrm{SO}_{2}$ by molten eutectic mixture of 


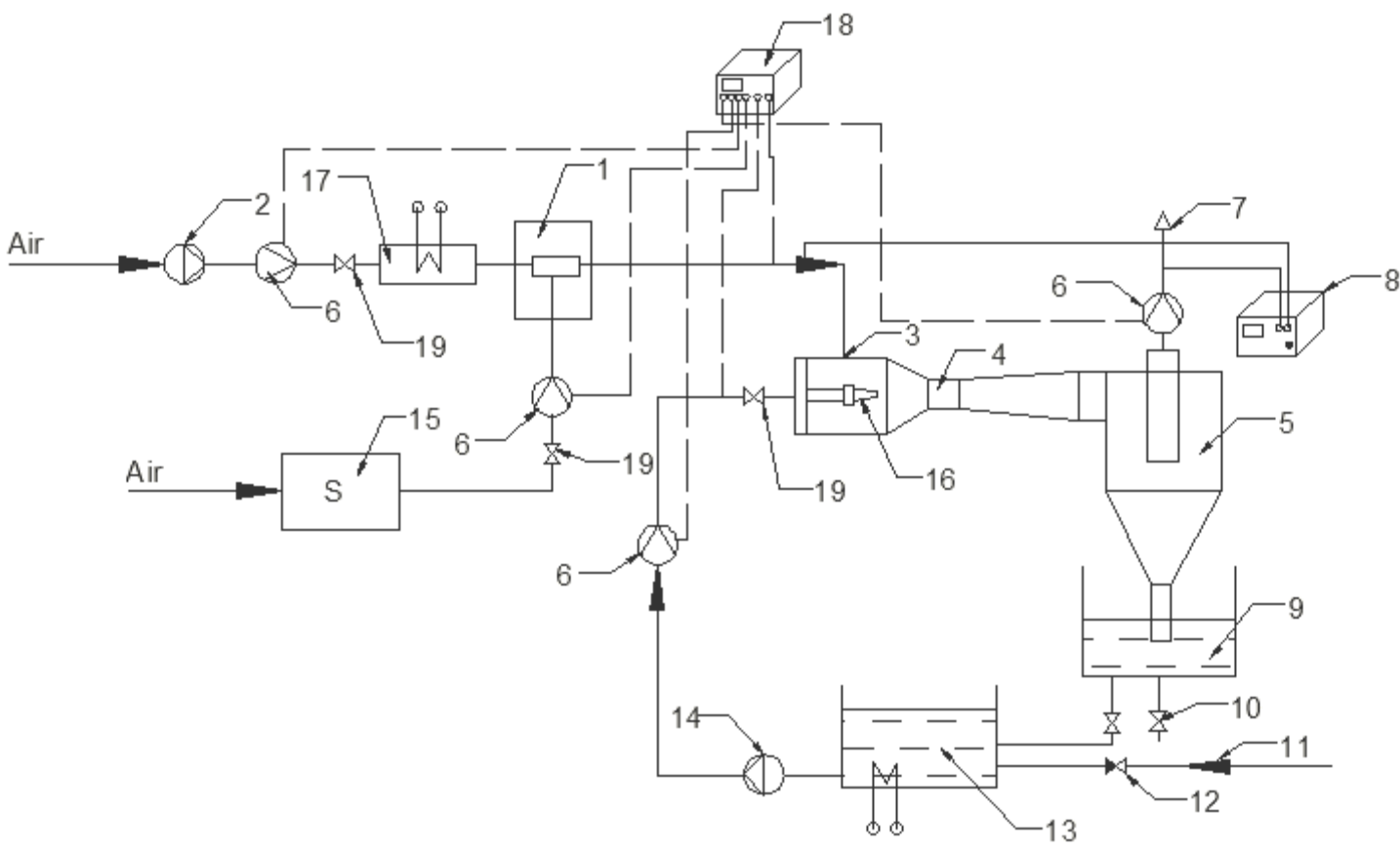

Fig. 1. Scheme of experimental setup

1 - injection unit; 2 - air blower; 3 - introduction of gas-air mixture into scrubber; 4 - Venturi scrubber; 5 - cyclone; 6 flow meter; 7 - output of purified gas mixture; 8 - gas analyzer; 9 - reservoir; 10 - valve; 11 - input of make-up absorbent;

12 - check valve; 13 - tank for absorbent;

14 - pump; 15 - furnace; 16 - nozzle; 17 - air heater; 18 - analogue-digital system of control and measurement; 19 adjusting valve.

carbonates of alkali metals (lithium, sodium and potassium) [9] is being used in practice.

The purpose of the work is creation of an experimental, rather uniform, installation for studying the effectiveness of catching sulfur oxides, nitrogen oxides and mercury vapors using the most active system of absorbent-absorbate which exceeds the absorptive capacity of the existing absorption pairs and reduces water consumption.

The principle scheme of the experimental installation for

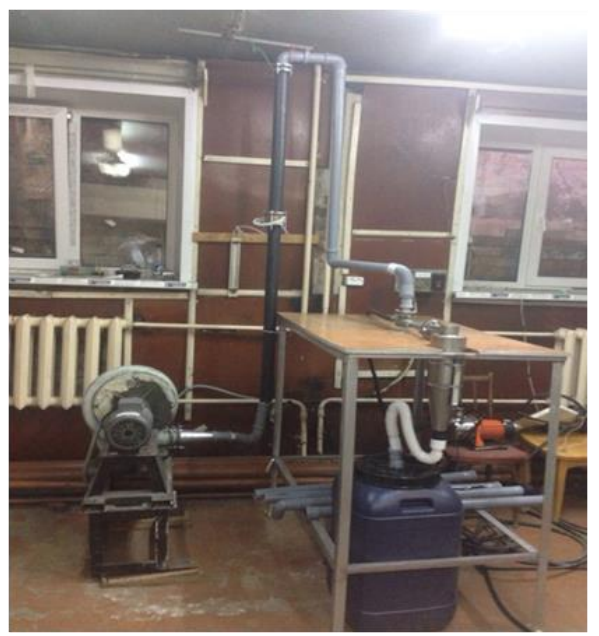

Fig. 2. Experimental setup studying the efficiency of catching sulfur and nitrogen oxides and mercury vapors by different absorbents based on [10] is shown in Fig. 1, and the picture of the installation is presented in Fig. 2.

The experimental installation consists of Venturi scrubber 4 , cyclone 5, furnace 15, air blower, pump, tanks, control devices, devices for measuring gas and water flow rates and temperatures and their concentrations in air. Air is supplied by the blower to injection unit 1 , where the injection of sulfur oxides or other gases from the furnace occurs; then, the formed gas mixture enters the Venturi scrubber, where oxides are absorbed by water with various additives. Then, the gaswater mixture passes through the cyclone, where it is separated into the gaseous and water components; water is discharged from the cyclone from the bottom into the receiving tank, gases are discharged from the top into the atmosphere. Gases and water flow rates are measured at the inlet to the scrubber; concentrations of gases in air are measured at the scrubber inlet and at the gas outlet from the cyclone. The gas and water flow rates can be changed by control valves; the studies can be carried out at various concentrations of impurities in air and different water flow rates, i.e. in a wide range of determining parameters. The data obtained can be used to plot the working and equilibrium lines, determine the coefficients of phase equilibrium, evaluate collection efficiency, and determine the most optimal regime 
of the installation operation on the chosen absorbent-absorbate pairs.

The mathematical model of absorption gas cleaning from harmful gas components (the absorption mathematical model AMM) developed in [11] [12] is adapted to chemisorption processes. The phase equilibrium coefficients for the absorbent- absorbate pair are determined by the experimental values of input and output parameters of the cleaned gas flow in the gas-cleaning device. Then, the inverse problem is solved using the obtained phase equilibrium coefficients for the irreversible process of chemisorption. For the found equilibrium coefficients, there is an opportunity to optimize the regime and geometrical parameters of the gas-cleaning devices depending on temperature and concentration of chemisorption additives in the absorbent solution. At the first stage of AMM-based research [12] [13], for the foam apparatuses (FA), the very idea was checked using experimental data [14] on the chemisorption process in a bubble column during $\mathrm{SO}_{2}$ extraction by the water solution of $\mathrm{NH}_{4} \mathrm{HCO}_{2}$. Results of $\mathrm{SO}_{2}$ extraction (experimental and computational) confirmed the correctness of the problem statement. Evaluation of consumption parameters of gases cleaned from $\mathrm{SO}_{\mathrm{x}}$ and $\mathrm{NO}_{\mathrm{x}}$ at TPP does not allow the use of FA as industrial gas-cleaning devices because of their limited capacity by gas volumes. For these purposes, the most suitable are the Venturi scrubbers (VS), widely used at thermal power plants as the units for cleaning gases from dust and harmful gas components. Gas velocities at the throat of Venturi tube (VT) can be up to $200-300 \mathrm{~m} / \mathrm{s}$. At the second stage of research, there was the task to adapt the AMM for the nozzle scrubbers and, in particular, for the VS in the chemisorption process in the framework of solving the inverse problem of finding the coefficients of phase equilibrium. The algorithms were worked out by calculations of $\mathrm{SO}_{2}$ absorption by water checked in [12].

The value of phase equilibrium coefficient within the framework of the inverse problem is defined as the solution to equation $\eta\left(m_{p x}\right)=\eta_{\text {ex. }}$, where $m_{p x} \eta$ is the coefficient of phase equilibrium, $\eta_{\text {ex. }}$ is the experimental value of effectiveness of target gas component extraction. The value of function $\eta\left(m_{p x}\right)$ for the argument $m_{p x}$ is defined by solving ordinary differential equations of AMM for the nozzle scrubbers. The model of VT with the following parameters was chosen as such a device: diameter of VS throat $d_{t}=0.02 \mathrm{~m}$, diffuser length $l=0.2 \mathrm{~m}$, diffuser apex angle $\alpha=6^{\circ}$. The parameters of convergent part are not important for calculation. These parameters will be used in the experimental installation with the air velocity in the VS throat of $80-100 \mathrm{~m} / \mathrm{s}$. The algorithm of numerical solution to the above equation consists of the following steps:

1) On the basis of arbitrarily given initial approximation $m_{p x}^{0}$, isolation interval for the root of equation $\left[m_{p x}^{\text {лев. }}, m_{p x}^{\text {прав. }}\right]$ was determined by the trial method;

2) Root $m_{p x}$ is defined by the method of half division with given accuracy $\varepsilon$;

3) The criterion of calculation completion is fulfillment of inequality

$$
\frac{m_{p x}^{\text {прав. }}-m_{p x}^{\text {лев. }}}{m_{p x}^{\text {прав. }}+m_{p x}^{\text {лев. }}}<\varepsilon .
$$

To check the efficiency of the algorithm, there were calculations using the theoretical values, obtained by solving the direct problem with given dependence of $m_{p x}$ (for $\mathrm{SO}_{2}$ ) on the temperature [12], as $\eta_{\mathrm{ex}}$ :

$$
m_{p x}=2976,58 T^{2}-1594158 T+215090898, \mathrm{~Pa} \text {. }
$$

The inverse problem (determination of efficient value of $m_{p x}$ ) was solved at $\varepsilon=10^{-5}$. Comparison of $m_{p x}$ values obtained by solving the inverse problem with dependencies used for solving the direct problem confirmed the efficiency of the algorithm. The direct problem was calculated with the obtained values of $m_{p x}$ and tabular data; it was found out that there were no differences in calculation results on thermodynamic parameters of the vapor-gas flow throughout the VS diffuser $\mathrm{SW}$, as well as in concentrations of $\mathrm{SO}_{2}$, dissolved in the droplets of dispersed water at absorption.

\section{RESULTS}

The algorithm for solving the inverse AMM problem was implemented numerically under the following boundary conditions for the Venturi scrubber at the diffuser inlet (tube throat):

- irrigation coefficient $q, \mathrm{~m}^{3} / \mathrm{m}^{3}-0.015$;

- initial droplet velocity $V_{k 0}, \mathrm{~m} / \mathrm{s}-4.0$;

- initial size of droplets $d_{k 0}, \mu \mathrm{m}-136.83$ (calculated by Nukiyama-Tanasawa formula, presented in $[11,12])$;

- initial temperature of droplets $T_{k 0}, \mathrm{~K}-333$;

- initial velocity of the gas-vapor flow $U_{0}, \mathrm{~m} / \mathrm{s}-80$;

- initial concentration of $\mathrm{SO}_{2} d_{10}, \mathrm{~kg} / \mathrm{kg}$ of dry air -0.1 ;

- initial moisture content $d_{\mathrm{n} 0}, \mathrm{~kg} / \mathrm{kg}$ of dry air -0.2 ;

- diameter of VS throat TB, m-0.02;

- diffuser length $l, \mathrm{~m}-0.2$;

- diffuser apex angle, degr. - 6;

- concentration of $\mathrm{SO}_{2}$ in droplet $c_{k 1,0}, \mathrm{~kg} / \mathrm{kg}$ of water -0 ;

- concentration of dust particles in the flow $C, \mathrm{~g} / \mathrm{m}^{3}-0$.

At the outlet of tube diffuser (the calculated value of the efficiency of $\mathrm{SO}_{2}$ extraction on the water droplets was set by solving the direct problem with approximation dependence of the tabular data for $m_{p x}$ (for $\left.\mathrm{SO}_{2}\right)$ ), in this case $\eta_{\mathrm{SO}_{2}}=$ 0.13491 .

According to the proposed algorithm, the effective value $\left(m_{p x}\right)_{\text {эф. }}=2278087.616 \mathrm{~Pa}$ was found for the given version along the entire length of the diffuser; it changes along the length from $1.9570 \cdot 10^{6}$ to $2.2911 \cdot 10^{6} \mathrm{~Pa}$, when solving the direct problem (Fig. 3).

Results of numerical solving of the direct problem for the tabular values of $m_{p x}$ and efficient value of $\left(m_{p x}\right)_{\text {эф. }}$ on $\mathrm{SO}_{2}$ extraction on water in the Venturi tube do not differ. At that, the efficiency of $\mathrm{SO}_{2}$ extraction under the given conditions is the same: $\eta=0.13491$. 


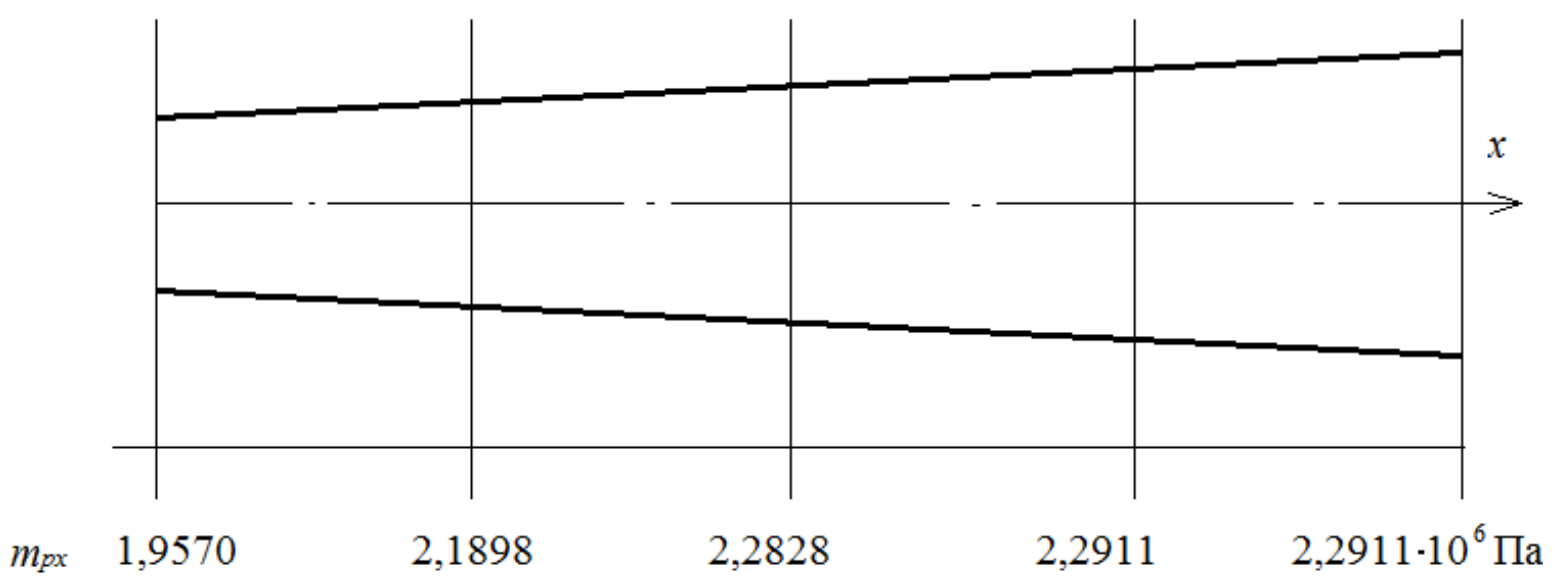

Fig. 3 - A change in $m_{p x}$ for $\mathrm{SO}_{2}$ along the diffuser of Venturi tube

To check the efficiency of the algorithm, there were calculations using the theoretical values, obtained by solving the direct problem with given dependence of $m_{p x}$ on the temperature [12], as $\eta_{\text {ex. }}$. The inverse problem (determination of efficient value of $m_{p x}$ ) was solved at $\varepsilon=10^{-5}$. Comparison of $m_{p x}$ values obtained by solving the inverse problem with dependencies used to solve the direct problem confirmed the efficiency of the algorithm. At that, the direct problem was calculated using the obtained values of $m_{p x}$ and tabular data; there were no differences in calculation results on thermodynamic parameters of the vapor-gas flow throughout the VT diffuser and concentrations of $\mathrm{SO}_{2}$ dissolved at absorption in dispersed water droplets.

At the initial stage of research, there were the tests on experimental installation performance at $\mathrm{CO}_{2}$ absorption by water. Tests on the experimental setup were carried out for the following constant parameters: absorbent (water) temperature of $6^{\circ} \mathrm{C}$; absorbate temperature of $22^{\circ} \mathrm{C}$; inert gas flow rate of $0.01697 \mathrm{~kg} / \mathrm{s}$; gas flow velocity in the Venturi scrubber throat of $44 \mathrm{~m} / \mathrm{s}$. Dependence of the amount of absorbed $\mathrm{M}$ component on irrigation density $q$ is shown in Fig. 4.

\section{DISCUSSION}

It can be concluded from the analysis that the proposed adaptation of the absorption model in the nozzle scrubbers to the processes of chemisorption for practical calculations is justified in terms of both the use of Henry's law for equilibrium parameters at the interface, and from the point of using the heat effect of absorption in the form of equation $r_{i}=\frac{R T^{2}}{M_{i}} \frac{d \ln m_{p x, i}}{d T}, \mathrm{~kJ} / \mathrm{kg}$, where $\mathrm{R}=8.314 \mathrm{~kJ} /(\mathrm{kmole} \cdot \mathrm{K})$ is the universal gas constant, $M_{i}$ is the molecular mass of the $i$-th gas component.

It should be noted that in the calculations the behavior and quantitative values of all thermodynamic parameters (sizes of droplet and "formations" (dust particles with condensate on the surface, temperatures of droplets, "formations", vapor-gas flow, concentrations of $\mathrm{SO}_{2}$ in droplets and "formation" condensate, and distributions of dust particle flows) differ insignificantly at $r_{i}$ and $10 r_{i}$.

This fact proves the ability to use the developed absorption model for obtaining the equilibrium coefficients for the

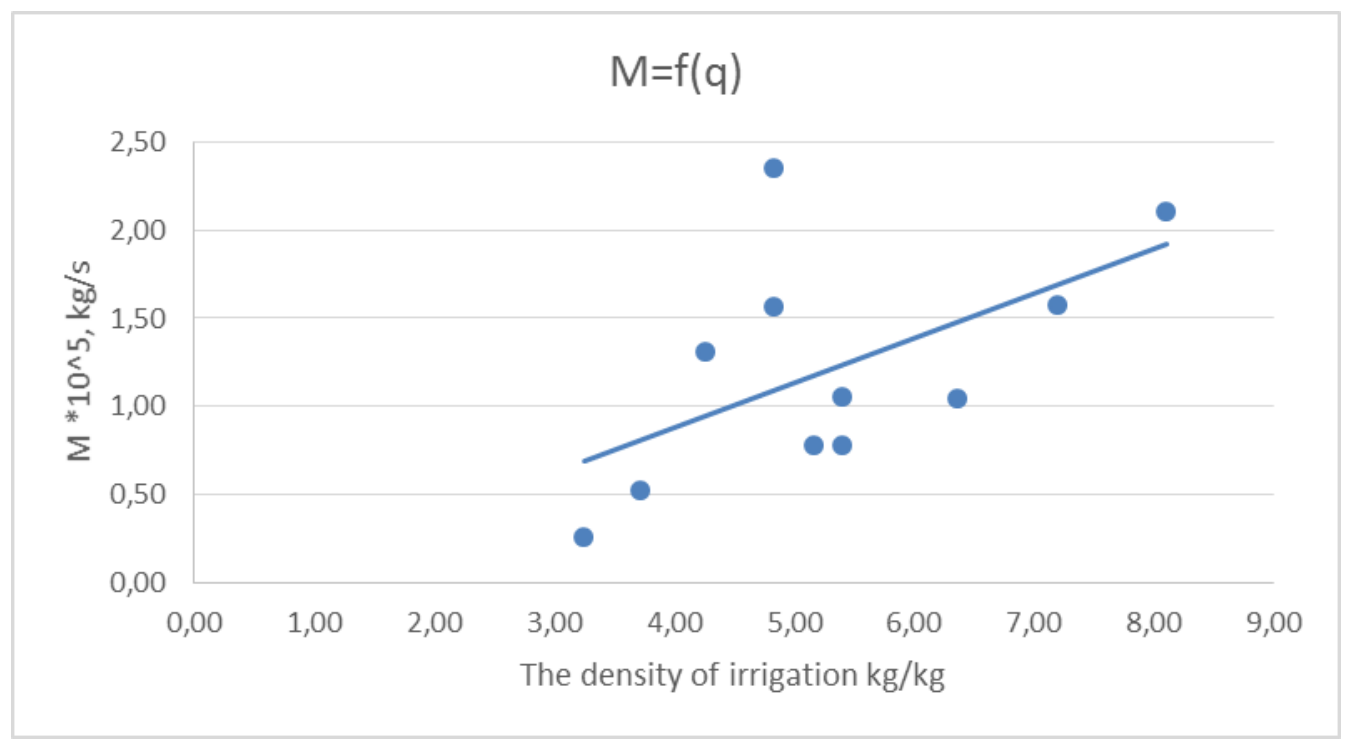

Fig. 4. Dependence of the amount of $\mathrm{CO}_{2}$ component absorbed from the gas-air mixture on irrigation density 
chemisorption process by solving the inverse problem with the test values of concentration of the target gas component and other thermal-physical parameters at the inlet and outlet of the experimental setup.

\section{CONCLUSIONS}

The proposed algorithm for solving the inverse AMM problem at the example of $\mathrm{SO}_{2}$ absorption on water in the model Venturi tube is numerically justified and can be used for experimental determination of the unknown coefficients of phase equilibrium of absorption for all absorbent-ascorbate pairs in the laboratory. There is reason to believe that this algorithm can be formally used to determine the effective equilibrium coefficients of chemisorption processes also for all absorbent-absorbate pairs by setting the experimental values of efficiency of target component extraction at the exit of the VS diffuser.

The experiments on carbon dioxide capturing have shown the possibility to use the installation for adaptation to chemisorption processes.

\section{ACKNOWLEDGEMENT}

The work was financially supported by the Russian Ministry of Education and Science in the framework of the Federal Target Program "Research and Developments in Priority Areas of Development of the Russian Scientific and Technological Complex for 2014-2020", by the Agreement No.14.583.21.0004 about the subsidies of June 16, 2014. The unique identifier of scientific research (project) is RFMEFI58314X0004.

\section{REFERENCES:}

[1] Belikov, S.E. Complex development of the methods for reducing emissions of nitrogen oxide at TPP by optimizing the burning processes and the ways of fuel combustion: Dissertation of the Doctor of Technical Sciences: 05.14.14, 03.00.16. - Moscow, 2006. - 282 p.

[2] Zvereva, E. R., Farakhov T.M., Iskhakov A.R. Reduction of harmful emissions of thermal power plants. KSEU Bulletin. 2011. Vol.8, No.1. P. 39-44.

[3] Dmitriev, A.V. Cleaning gas emissions at TPP, operating on liquid and solid fuel in the apparatuses of the vortex type; Dissertation of the Candidate of Technical Sciences: 05.14.14 - Kazan, 2006. - 140 p.

[4] Zykin, Yu.V. Development of resource-saving technology of wet cleaning of TPP flue gases from ash: Dissertation of the Candidate of Technical Sciences: 05.14.14. - Kazan, 2003. - 107 c.

[5] Kazaryan, A.S. Complex cleaning the flue gases of heat-generating installations: Dissertation of the Candidate of Technical Sciences: 05.23.03 - Rostov-on-Don, 2005. 128 p.

[6] Ramm, V.M. Absorption of Gases. Moscow: "Khimiya", 1976. 656 p.

[7] Volchin, I.A., Mezin S.V., Rudenko L.N., Yasinetsky A.A. Investigation of sulfur dioxide absorption in carbamide solution. Energetik. 2015. \# 6. P. 24-26.

[8] Kulish O.N., Kuzhevatov S.A., Gleizer I.Sh., Orlova M.N., EIvanova.V., Zykov A.M., Bragina O.N., Kolchin K.I., and Anichkov S.N. Increasing the efficiency of flue gas cleaning from nitrogen oxides in SNCR installations. Energetik. - 2014. - No. 4. - P. 31-34.

[9] Dosmukhamedov N.K., Kaplan V.A., Zholdasbayev E.E., Dosmukhamedov D.N., Lubomirski I. Development of the method for flue gas cleaning from sulfur at thermal coal power plants. Coal. 2015. August. P. 106-110

[10] Kalachev A.I. Principle scheme of the exhaust gas purification at coal power plants for generation of high-quality by-products of coal combustion. TPC. Development Strategy. - 2015. - No. 4 (39). - P. 3641.

[11] Shilyaev M.I., Khromova E.M. Modeling of heat and mass transfer and absorption-condensation dust and gas cleaning in jet scrubbers. Mass Transfer - Advances in Sustainable Energy and Environment Oriented Numerical Modeling, Edited by Hironori Nakajima. - Vienna, Austria: In Tech, 2013. - PP. 163-194.

[12] Shilyaev M.I., Khromova E.M., Tolstykh A.V.Contact heat and mass transfer in the nozzle and bubble apparatuses. Modeling, optimization of heat and mass transfer and absorption-condensation dust and gas purification. Germany: LAP LAMBERT Academic Publishing, 2012. 273 p.

[13] Shilyaev M.I., Tolstykh A.V. Modelling of gas absorption processes in bubblers. Thermophysics and Aeromechanics. - 2013. - Vol.20, No.5. P. 565-576.

[14] Xiaolei, Li Mass transfer of SO2 absorption with an instantaneous chemical reaction in a bubble column / Li Xiaolei, Zhu Chunying, Lu Sumin, Ma Younguang // Brazilian Journal of Chemical Engineering. 2013. - Vol. 30, № 03. - Pp. 551-562. 